\title{
LOW-TEMPERATURE PROPERTIES OF TWO INTERACTING MAGNETIC IMPURITIES
}

\author{
P. SCHLOTTMANN ${ }^{\star}$ and J.W. RASUL ${ }^{b}$ \\ "Department of Physics, Temple University, Philadelphia, Pennsylvania 19122, USA \\ ${ }^{b}$ Department of Physics, University of Michigan, Ann Arbor, Michigan 48109, USA
}

\begin{abstract}
We discuss a simple model consisting of four spins $\frac{1}{2} ; S_{1}$ and $S_{2}$ representing the impurity spins and $s_{1}$ and $s_{2}$ the conduction electron spin-densities at the impurity sites, coupled by three antiferromagnetic couplings: $T_{K}$ describing the local Kondo singlet-triplet splitting, $J$ is the RKKY and $K$ is a cross coupling between $S_{1}\left(S_{2}\right)$ and $s_{2}\left(s_{1}\right)$. This model has a critical point at $J=2 \mathrm{~K}=2 T_{\mathrm{K}}$ with similar properties as the unstable fixed point recently discovered by Jones, Varma and Wilkins: (i) a specific heat anomaly, (ii) a well-behaved uniform magnetic field susceptibility, (iii) a divergent staggered susceptibility, and (iv) a strongly varying expectation value $\left\langle\boldsymbol{S}_{1} \cdot \boldsymbol{S}_{2}\right\rangle$ with an average value of $-\frac{1}{4}$.
\end{abstract}

Any attempt to understand the nonuniversal behavior of heavy fermion compounds must involve at least two competing energy scales. One of them, the Kondo temperature $T_{\mathrm{K}}$, is the energy scale on which an isolated magnetic moment is screened (spincompensated) by the conduction electron spin-density. The origin of the other energy scale(s) are the interactions between the magnetic moments and the interference of the screening clouds at different sites.

The simplest model showing the competition between two energy scales is the two-impurity Kondo model. The two energy scales in this case are $T_{\mathrm{K}}$ and the Ruderman-Kittel-Kasuya-Yosida (RKKY) interaction, whose dependence on $k_{\mathrm{F}} R$ yields the desired nonuniversal behavior. The Hamiltonian is given by

$$
\begin{aligned}
& \mathscr{H}=\sum_{k \sigma r} \epsilon_{k} C_{k \sigma}^{\dagger} C_{k \sigma}+J \sum_{\substack{k, k^{\prime}, l \\
r, \sigma r^{\prime}}} \\
& \left.\times \boldsymbol{S}_{l} \cdot C_{\boldsymbol{k} \sigma \sigma}^{\dagger} \boldsymbol{s}_{\sigma \sigma}, C_{\boldsymbol{k} J^{\prime}} \exp \left[-\mathrm{i}\left(\boldsymbol{k}-\boldsymbol{k}^{\prime}\right) \boldsymbol{R}_{l}\right)\right],
\end{aligned}
$$

where $J$ is the exchange parameter, $R=\left|\boldsymbol{R}_{\mathbf{1}}-\boldsymbol{R}_{2}\right|$, $l=1,2$ labels the impurities, $S_{l}$ are the impurity spins and $2 s$ the vector of Pauli matrices. The RKKYinteraction is generated in second and higher order perturbation in $J$. The two-impurity model has been treated within various approaches [1], including the $1 / N$-expansion [2].

The groundstate of the two-impurity system is a singlet. A formal Fermi-liquid expansion [3] shows that the relation between the specific heat $\gamma$-coefficient and the spin susceptibility is nonuniversal. The renormalization group approach (poor-man's scaling [4] and Wilson's numerical one [5]) yields that for strong ferromagnetic RKKY coupling between the impurities. their spins lock into a triplet state, which at low $T$ is Kondo compensated in analogy to the twochannel Kondo problem. For strong antiferromagnetic RKKY coupling, on the other hand, the spins of the two impurities compensate each other and the Kondo effect only plays a secondary role. Separating the regions of attraction of these two stable $T=0$ fixed points, is necessarily a third, unstable, fixed point with highly unusual properties discovered by Jones, Varma and Wilkins [6]. At this fixed point the staggered field response diverges, tracking the specific heat coefficient $\gamma$, while the homogeneous-field susceptibility remains finite and the expectation value $\left\langle\boldsymbol{S}_{1} \cdot \boldsymbol{S}_{2}\right\rangle$ is approximately -0.25 for widely separated values of $T_{\mathrm{K}}$. Denoting with $J$ the (antiferromagnetic) RKKY-coupling strength this unstable fixed point occurs at $J / T_{\mathrm{K}} \simeq 2.2$ [6].

In order to gain a phenomenological understanding of the unusual properties of the unstable fixed point we proposed a simple model in a recent comment [7]. The purpose of this paper is to present more details of our calculation. Other attempts to explain the intriguing properties of the unstable fixed point can be found in refs. $[8,9]$.

We first briefly recall the picture arising from the renormalization group treatment of the single impurity. The degrees of freedom of the electron gas are gradually eliminated, yielding a singlet groundstate formed by the impurity spin $S=\frac{1}{2}$ and the conduction electron spin-density $s=\frac{1}{2}$. At the $T=0$ fixed point the interacting impurity can be represented by $H=$ $T_{\mathrm{K}} S \cdot s$, leading to a singlet groundstate $(|S\rangle)$ and a triplet $(|T m\rangle, m=+, 0,-)$ with excitation energy $T_{\mathrm{K}}$. The susceptibility is given by the field-induced transitions between the singlet and the triplet and is of the order of $1 / T_{\mathrm{k}}$. 
Our simple model for two interacting impurities is based on this picture. It consists of four spins $\frac{1}{2}: S_{1}$ and $S_{2}$ represent the impurity spins and $s_{1}$ and $s_{2}$ denote the conduction electron spin-densities at the impurity sites. All other degrees of freedom of the electron gas are neglected in the same spirit as for the single impurity. The four spins are coupled by three antiferromagnetic couplings: $T_{\mathrm{K}}$ again phenomenologically describes the energy splitting between the $T=0$ Kondo ground-singlet and excited-triplet states, $J$ is the ususal RKKY coupling, and $K$ is a cross-coupling between $S_{1}\left(S_{2}\right)$ and $s_{2}\left(s_{1}\right)$,

$$
\begin{aligned}
\mathscr{H}= & T_{\mathrm{K}}\left\{\boldsymbol{S}_{1} \cdot \boldsymbol{s}_{1}+\boldsymbol{S}_{2} \cdot \boldsymbol{s}_{2}\right\}+J \boldsymbol{S}_{1} \cdot \boldsymbol{S}_{2} \\
& +K\left\{\boldsymbol{S}_{1} \cdot \boldsymbol{s}_{2}+\boldsymbol{S}_{2} \cdot \boldsymbol{s}_{1}\right\}
\end{aligned}
$$

This model contains the same type of effective interactions (already representing the many-body processes) as the fixed-point Hamiltonian proposed in [4].

The sixteen eigenstates of the Hamiltonian can be classified according to the total spin $S$, its $z$-component and the parity (with respect to permutations of the impurities) of the wave function. A convenient basis of states is the direct product of the Kondo singlet $\left|S_{1}\right\rangle$ and triplet $\left|T_{1} m\right\rangle$ of impurity 1 with those of impurity 2 . The direct product of the two Kondo triplets yields an even $S=2$ multiplet, and odd $S=1$ triplet and a singlet of even parity; the product of the Kondo triplet at site 1(2) with the Kondo singlet of site 2(1) can be combined into two triplets of even and odd parity, respectively, while the direct product of the two singlets yields an even singlet. States with equal quantum numbers (parity, $S, S_{z}$ ) are admixed by the Hamiltonian (2), such that the largest matrix to be diagonalized is $2 \times 2$ (for singlets and odd triplets). The energies of the eigenstates are

$$
\begin{aligned}
& E=\frac{T_{\mathrm{K}}}{2}+\frac{J}{4}+\frac{K}{2} \\
& E=-\frac{T_{\mathrm{K}}}{2}+\frac{J}{4}-\frac{K}{2} \\
& \text {, }(S=1 \text {, even }) \\
& E=-\frac{J}{4} \pm \frac{1}{2}\left[J^{2}+\left(T_{\mathrm{K}}-K\right)^{2}\right]^{1 / 2} \\
& \text {, }(S=1, \text { odd }) \\
& E--\frac{T_{\mathrm{K}}}{2}-\frac{J}{4}-\frac{K}{2} \pm\left[T_{\mathrm{K}}^{2}+\frac{J^{2}}{4}+K^{2}\right. \\
& \left.-\frac{J K}{2}-\frac{J T_{\mathrm{K}}}{2}-T_{\mathrm{K}} K\right]^{1 / 2} \\
& \text {, }(S=0 \text {, even })
\end{aligned}
$$

The low $T$ properties of this model are now discussed for antiferromagnetic couplings $J, K$ and $T_{\mathrm{K}}$.

For $J, K, T_{\mathrm{K}}>0$ the groundstate is a singlet (6), except along the line $K=T_{\mathrm{K}}$ for $J \geqslant 2 T_{\mathrm{K}}$, where the lower-lying odd triplet (5) is degenerate with the singlet. At the point $\mathrm{P}$ defincd by $J=2 K=2 T_{\mathrm{K}}$ the degeneracy is fivefold, since the two singlets are degenerate with the odd triplet. This singular point $P$ forms the basis of our further discussion.

In order to understand the nature of the degeneracies we first discuss the groundstate expectation value of $S_{1} \cdot S_{2}$ along the line $K=T_{\mathrm{K}}$. The groundstate energy is $E_{\mathrm{G}}=-3 J / 4$ for $J>2$ and $E_{\mathrm{C}_{\mathrm{t}}}=-2 T_{\mathrm{K}}+J / 4$ for $J<2$. Using that $\left\langle S_{1} \cdot S_{2}\right\rangle=\partial E_{\mathrm{G}} / \partial J$ we obtain that $\left\langle S_{1} \cdot S_{2}\right\rangle=-\frac{3}{4}$ for $\left.J\right\rangle 2$, i.e., the impurity spins are locked into a singlet state, and $\left\langle S_{1} \cdot S_{2}\right\rangle=\frac{1}{4}$ for $J<2$, i.e, the impurity spins form a triplet.

The impurity singlet for $J>2$ implies that the fourfold degeneracy of the Hamiltonian is due to the spin-densities of the conduction electrons, which along the line $K=T_{\mathrm{K}}$ are then completely decoupled from the impurities. The degeneracy is then an artifact of the simplicity of the model and would be quenched by the (neglected) motion of the conduction electrons. For $J>2$ this line has now the properties of the antiferromagnetic fixed point of the full two-impurity Hamiltonian.

The impurity triplet, on the other hand, for $J<2$ along the line $K=T_{\mathrm{K}}$ is spin-compensated by the conduction electron spin-densities, since the groundstate of the Hamiltonian (2) is a singlet. This resembles the properties of the other stable fixed point of model (1), where the impurity spins first lock into a triplet and are then spin-compensated in analogy to the two-channel Kondo problem.

These two regions of the line $K=T_{\mathrm{K}}$ are separated by the singular point $\mathrm{P}$ at $J=2 T_{\mathrm{K}}$. At $\mathrm{P}$ the two singlets and the lower-lying odd triplet all have energy $E=-1.5 T_{\mathrm{K}}$, the even triplet has energy $E=-0.5 T_{\mathrm{K}}$, the other odd triplet $E=0.5 T_{\mathrm{K}}$ and the $S=2$ multiplet $E=1.5 T_{\mathrm{K}}$. The high symmetry at $\mathrm{P}$ is a consequence of the frustration of the spins. Below we argue that $P$ resembles the unstable fixed point of model (1) found in ref. [6].

The singlet wave functions correspond in general to linear combinations of (a) $\left|S_{1}\right\rangle \otimes\left|S_{2}\right\rangle$, the Kondo singlets at each site, and (b) the antiferromagnetically correlated impurities $3^{-1 / 2}\left[\left|T_{1}+\right\rangle \otimes\right.$ $\left.\left|T_{2}-\right\rangle-\left|T_{1} 0\right\rangle \otimes\left|T_{2} 0\right\rangle+\left|T_{1}-\right\rangle \otimes\left|T_{2}+\right\rangle\right]$. At the singular point $\mathrm{P}$, however, the mixing vanishes and the dominant character of the wave function changes abruptly between (a) and (b); the magnitude of the 
discontinuity depends on the way the point $\mathrm{P}$ is approached in parameter space.

The properties close to the singular point $P$ are the following: (i) the degeneracy of the groundstate gives rise to a specific heat anomaly, which, when coupled to the electron gas continuum, would produce a nonanalytic behaviour of $\gamma$ at $\mathrm{P}$, but $\gamma$ is well-defined elsewhere. (ii) A uniform magnetic field couples the singlets to the even parity triplet, leading to a susceptibility $x \sim 1 / T_{\mathrm{k}}$ which is always finite and has a small cusp at $P$, but otherwise no irregular behavior. (iii) However, a staggered field admixes the odd-parity triplets with the singlets, giving rise to a divergent staggered susceptibility $\chi$, at $\mathbf{P}$ (Curie law). This divergence is controlled by the square roots in (5) and (6) and $\chi_{\mathrm{s}}$ is dramatically reduced even for small deviations from $P$. (Note that a staggered field acting on the impurites does not couple the degenerate groundsinglet and triplet along the line $K=T_{\mathrm{K}}, J>2$, although this is permitted by symmetry.) (iv) $\left\langle S_{1} \cdot S_{2}\right\rangle$ varies discontinuously across the singular points as a consequence of the abrupt change of dominant character of the singlet wavefunctions. At $\mathrm{P},\left\langle\boldsymbol{S}_{1} \cdot \boldsymbol{S}_{2}\right\rangle=\frac{11}{20}$ due to the fivefold degeneracy, but the half-sum across the discontinuity is -0.25 as it can easily be verified using $\partial E / \partial J$ in eq. (6). The discontinuity may resemble the strong variation of $\left\langle S_{1} \cdot S_{2}\right\rangle$ with $J / T_{\mathrm{K}}$ across the unstable fixed point found in ref. [6]. Coupling to the electron gas continuum would smear the discontinuity. (v) All these properties occur for $J / T_{\mathrm{K}}=2$, as compared to 2.2 for the unstable fixed point in ref. [6].

In spite of the simplicity of our model, we recover at the singular point most of the anomalous properties of the unstable fixed point discovered in ref. [6]. Our brief calculation may suggest insights into the compli- cated nature of the interference between Kondo screening and intersite spin correlations.

We would like to thank Dr. B.A. Jones for corrections and valuable comments. This work was supported by DOE Grant No. DE-FG02-87ER45333.

\section{References}

[1] S. Alexander and P.W. Anderson, Phys. Rev. 133 (1964) A 1594 .

S.B. Caroli, J. Phys. Chem. Solids 28 (1967) 1427.

M.T. Beal-Monod, Phys. Rev. 178 (1969) 874.

K. Sato and Y. Nagaoka, Prog. Theor. Phys. 47 (1972) 348.

K. Yamada, Prog. Theor. Phys. 62 (1979) 901.

M. Lavagna, J. Magn. Magn. Mat. $47 \& 48$ (1985) 360.

[2] J.W. Rasul and A.C. Hewson, Solid State Commun. 52 (1984) 217.

I. Rrandt, H. Keiter and F.-S. Liu, Z. Phys. B 58 (1985) 267.

P. Coleman, Phys. Rev. B 35 (1987) 5072

[3] P. Schlottmann, Phys. Rev. B 21 (1980) 1084, B 34 (1986) 2007

[4] C. Jayaprakash, H.R. Krishna-Murthy and J.W. Wilkins. Phys. Rev. Lett. 47 (1981) 737

[5] B.A. Jones and C.M. Varma, Phys. Rev. Lett. 58 (1987) 843.

[6] B.A. Jones, C.M. Varma and J.W. Wilkins, Phys. Rev. Lett. 61 (1988) 125

[7] J.W. Rasul and P. Schlottmann, Phys. Rev. Lett. 62 (1989) 1701.

[8] B.A. Jones, B.G. Kotliar and A.J. Millis, Phys. Rev. B 39 (1989) 3415.

[9] B.A. Jones and C.M. Varma, Phys. Rev. B 40 (1989) 324. 